\title{
Wireless Energy Harvesting in Internet-of-Things Communication Systems with Optimized Energy Efficiency using PSO
}

\author{
Rekha, Ritu Garg
}

\begin{abstract}
IoT is an emerging technology having a wide range of application areas. IoT applications are also affecting human lives. But these small devices are battery powered which is major problem for IoT systems. Wireless energy transfer is a good solution for such systems. Both information and energy can be transmitted together by wireless energy. In this paper, time splitting-based relaying (TSR) protocol is used by relay node to harvest the energy in IoT system. Here, dual-hop IoT system is considered for analysis. System with three different Wi-Fi protocols is examined against the energy efficiency at the destination node. All three protocols are analysed individually. Further, Particle Swarm Optimization (PSO) technique is used to optimize the energy efficiency of the considered IoT system.
\end{abstract}

Index Terms: Energy harvesting, Internet of things, Time splitting based relay protocol, Wireless energy.

\section{INTRODUCTION}

Internet of Things (IoT) is an intelligent worldwide network of uniquely addressable things for sharing data and performing complex tasks [1]-[2]. Things in IoT perspective is extremely sweeping such that it can be environmental elements, wireless devices, sensors, actuators, Radio-Frequency IDentification (RFID) tags and individual objects like tablets, smart watches, cameras, cell phones, etc. $[3,4]$. IoT devices possess some characteristics like low power consumption, sensing/actuating method, communication and small size [5]. Internet of Things" was introduced by Kevin Ashton in 1998 in the area of wireless communication and networking [6]. IoT is defined in several ways by many researchers. Some of them are:

-IoT is a 3A idea which means anytime any media can be connected to anywhere" [7].

-IoT represents an interaction between the two world, physical and digital, using a lot of actuators and sensors" [8].

-IoT can be defined as embedded networking and computing capacities in different types of object" [9].

As per IDC study, in 2020 predicted spending on IoT will reach to $\$ 1$ trillion [10]. Fig 1 illustrates the worldwide growth of connected devices [11]. These connected devices will help to develop the smart environment by monitoring, managing, analyzing and taking intelligent decisions. IoT has already used in many sectors for surveillance,

\section{Revised Manuscript Received on July 02, 2019}

Rekha, Computer Engineering, NIT Kurukshetra, Kurukshetra, India.

Ritu Garg, Computer Engineering, NIT Kurukshetra, Kurukshetra, India.

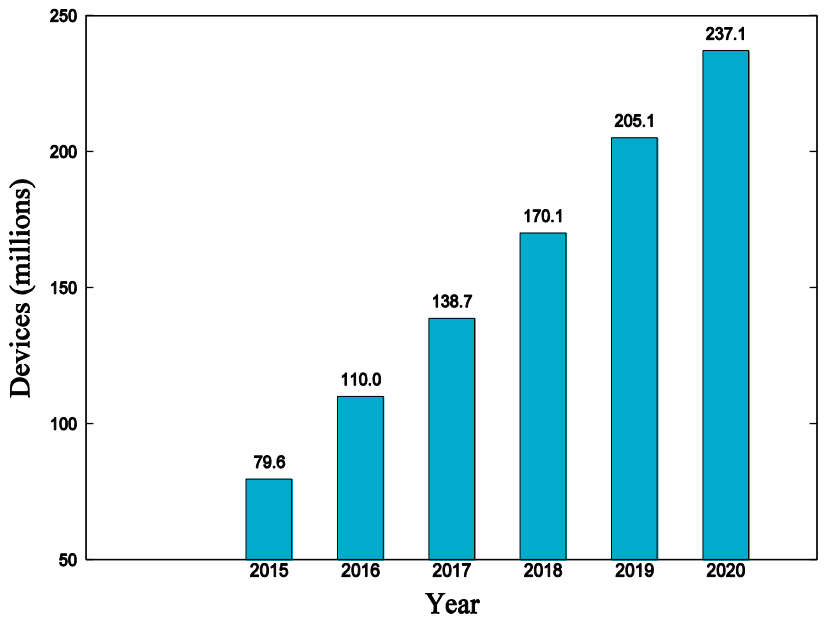

Fig 1 Growth in Number of Connected Devices

decision-making etc. Application domains of IoT are Transport, Health, Smart City, Home Appliances, E-governance, Logistics, Agriculture, Automation, Smarts grids, Supply Chain Retail, Industries (like Aerospace and Aviation, Pharmaceutical, Telecommunications) and many more $[12,13]$. Various IoT application domains are shown in Fig 2 . IoT is rapidly growing day by day.

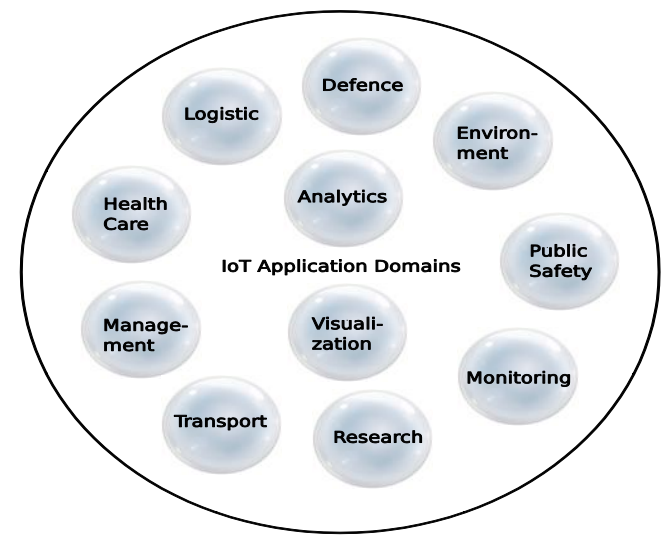

Fig 2 IoT application domains

Today, IoT is an emerging technology and it is modifying the world we live in by progressing toward to interconnected model. Key feature of IoT devices is that they are battery powered. Due to small size of connecting devices, there is

size constraint on battery 
also. Battery need to be replaced or recharged after a time duration. Many applications need sensors to be deployed in the unwelcoming territories. Though, replacement of battery is not impossible in these situations. But it is difficult task and sometimes it costs even more than the device's cost. Therefore, providing the sufficient energy to the IoT devices to operate is the prepotent obstacle in IoT networks.

For powering a significant number of devices over a long duration of years, energy harvesting $(\mathrm{EH})$ is most promising solution. Where battery replacement is frequent and tedious task, $\mathrm{EH}$ has a potential to power the devices for a long duration. $\mathrm{EH}$ is process of scavenging energy form the ambient sources which are always available. Harvested energy need to be converted into electric energy before use. Due to renewable energy sources, EH is gaining attention currently. Different types of renewable energy exist in surrounding like solar, wind, heat, mechanical, geothermal, hydro-power, radio frequency (RF) etc [14]. Employment of EH techniques can recharge the battery of node or can directly provide energy for operation. In both the cases, it will enhance the lifetime of both node and network. Therefore, Energy harvesting based designs are becoming more fascinating.

Recently, EH networks have attracted a lot of attention in literature. Wireless EH techniques can be used to recharge batteries and thus enhance the network's lifetime [15]. Many studies in the literature have proposed techniques for $\mathrm{EH}$ in various application domains of IoT system [16]. Gorlatova et al. [17] focus the human motion thoroughly and characterize acceleration traces to harvest the kinetic energy for the practical IoT nodes. For increasing the efficiency, a storage-less and converter-less solar EH system is discussed in [18]. This system uses a PMU to control the PV voltage and also can perform MPPT tracking resulting in improved efficiency. Also, EH techniques can be integrated with the energy efficient algorithms. Such a integration work is presented in paper [1]. Here, EH is integrated with adaptive energy efficient technique (ABSD) has been proposed for WSN-enabled applications which harvest the roadside kinetic energy. Depending on the traffic patterns and remaining energy, sleeping period is changed. During the sleeping time, node harvests the energy for charging. EH techniques are also used in the IoT based healthcare systems.

Babar et al. [19] point towards the efficacy of EH in healthcare. Regarding this, a conceptual framework for EH is discussed with big data processing in IoT enabled health monitoring. Its not only single type of energy that can be harvested by a particular system. More than one ambient energy can be harvested. Hybrid energy harvesting in IoT systems described by Vaisband [20]. This 3D heterogeneous platform can harvest the four different types of ambient energy (i.e. Electro Magnetic, Solar, Thermal and Kinetic) individually.

Over the other energy harvesting techniques, wireless energy harvesting has a major benefit. This is only method which is capable of carrying both information and energy simultaneously. Node can obtain both energy for communication and information to process from the same received signal. The received signal can be divided into two parts. One can be used to serve as energy source and remaining can be used to acquire information. Many researchers worked with wireless energy harvesting [21]-[23].

This division can affect the performance of system. As the power transmitted by the source is both energy and information source for the receiver. So it should be considered how much amount of power should be transmitted by source node. So it is required to optimize the transmitted power to maximize the system performance.

In this paper, a dual-hop relay system is considered in which energy efficiency at the destination node is optimized using PSO. Here, energy efficiency is characterized as the ratio of spectrum efficiency to the total power expenditure of the complete IoT network. To the best of my knowledge, most of the research work in this area considered numerical analysis methods to optimize energy efficiency which requires high computation overhead. However, we have used PSO to maximum energy efficiency by varying the power transmitted by source node that provides fast convergence with small computation overhead. We have optimized energy efficiency of IoT system with three Wi- Fi protocols 802.11 $\mathrm{b} / \mathrm{g} / \mathrm{n}$.

\section{Motivation}

In today's connected world, everyone is expecting that almost all the objects from small watches to large size vehicles are connected to the internet. Most of these objects are small size wireless sensors. Major drawback of these IoT devices is that these are mostly powered through batteries. And key obligation is sensors are deployed in all type of areas. Therefore, powering the sensor by small batteries for a long duration is not a significant solution. Due to small size batteries, provision of power for long functional duration puts a limitation on the IoT devices. So, researchers have been thinking to acquire environmental energy for the IoT device. After collecting the surrounding energy, it can be directly used by the node or can be stored for future use. It may or may not possible to obtain ambient energy when it is needed. This can happen due to unavailability of energy in environment (e.g. solar energy is not available at night and cloudy days). Hence, there is strong need to store energy for later use. For storage purpose batteries, supercapacitors etc. can be used.

\section{BACKGROUND}

Among the different kinds of renewable energy: solar, wind, mechanical, electromagnetic, thermal, geothermal, RF, etc., harvesting energy from radio frequency (RF) signals has captured significant attraction in literature recently $[15,24$, 25]. RF EH is a wireless energy transfer technique which uses the radio signals having frequency within the range from $3 \mathrm{kHz}$ to $300 \mathrm{GHz}$. Unlike other wireless techniques, RF EH has no limitation. RF energy conversion efficiency varies with input power level, however it is small when small amount of power is harvested [25].

Biggest advantage of using RF signal for energy harvesting over other techniques is that RF signal can carry both information and energy simultaneously. Dual use of RF signal for transporting energy and information both at a time has been proposed first in [26]. Authors study basic performance trade-off for transmitting power and information simultaneously. Unlike [26], in which authors consider 
point-to-point system with single antenna for study,

in [27] authors consider a multi-input multi-output relay system to study the various trade-off for simultaneously information and energy transmission to optimize the efficiency.

Two protocols, Time Switching (TS) and Power Splitting (PS), are discussed in [28] for energy harvesting receiver. In TS based protocol, relay node switch between energy harvesting phase and information processing phase according to time. However, in PS based protocol, relay splits the received power for energy harvesting and information processing phase. Using mechanisms TS and PS discussed in [28], the relaying protocols Time Switching based Relaying (TSR) and Power Splitting based Relaying (PSR) are proposed in [29]. In this, authors consider a one-way communication network having an amplify-and-forward (AF) relay. Performance of system is checked against the system throughput. Conclusion derived is that for high value of transmission rate, small EH efficiency and small value of SNR (signal-to-noise-ratio), TSR protocol performs better than the PSR protocol and vice versa.

Tang et al. [30] use the TSR and PSR protocols for transmitting energy and signal together in wireless acoustic sensor network. Performance of both protocols is analysed based on the throughput efficiency and results show that to achieve higher transmission rate at secondary receiver, PSR protocol outperforms TSR at low transmission power. Unlike [28]-[30], Tariq et al. [31] consider relay network based on two-way amplify-and-forward (AF). TSR and HPTSR (hybrid power-time switching based relaying protocol) protocols are analysed for harvesting at relay node. For above discussed network, HPTSR performs better that TSR and PSR at high SNR and low transmission rate. But TSR outperforms HPTSR and PSR at high transmission rate and low SNR. Baofeng et al. [32] consider the PSR protocol for transferring information and energy at the same time in IoT enable wireless communication. Dual hop IoT system is considered in which first relay harvest the energy and then it transfers power and information to the respective users. Nguyen et al. [33] examined the performance of relay system with energy harvesting. In this paper, system is proposed with full duplex and energy harvesting. Performance of proposed system is analysed in terms of outage probability and symbol error probability with energy harvesting both at source and relay nodes. Bachi et al. [22] analyse the throughput of cognitive radio using numerical method.

In present paper, the harvested energy by relay node is analysed for relaying node in IoT based wireless communication system. Both power and information are transmitted at the same time. Performance is measured in terms of energy efficiency which is ratio of the throughput over total power consumed. Energy efficiency at the destination is optimized using PSO.

\section{Proposed System}

Wireless communication system is considered as shown in the Fig 3. In this dual hop system, wireless information and energy transmit from source node to destination node. This transmission is through the relay node. Relay node acquires the wireless information for the time period T. For some fraction of time period $\mathrm{T}$, relay node harvests the energy from received wireless energy and for rest of the time period it will process the information.

For the system model in present paper, we made following assumptions:

1. IoT wireless communication system is dual hop for simplicity. From source to destination, there is no direct communication. Communication is through only relay node.

2. During first proportion of $\mathrm{T}$, relay node harvest the energy and later duration is used to forward the information from source to destination.

Fig 4 represents the system model for the energy harvesting and information processing based on the time. $\mathrm{T}$ is the time period required to transfer the certain amount of information from source to destination. $\alpha_{h}$ is fraction of time period and for time duration for which relay node harvest the energy where $0 \leq \alpha_{h} \leq 1$. For rest of the time period i.e. $\left(1-\alpha_{h}\right) \mathrm{T}$, information is being processed. Each Time block $\left(1-\alpha_{h}\right) \mathrm{T} / 2$ is used to transfer the information from source to relay and relay to destination respectively. During the energy harvesting phase, relay node harvest the energy and consumes this energy during transmission phase. Signal received at relay is $S_{r}^{r}$ expressed as

$$
S_{r}^{r}=\frac{\sqrt{P_{s}} h s}{\sqrt{d_{s r}^{m}}}+n_{r}^{s}
$$

where, $\mathrm{s}$ is transmitted signal with unit power, $\mathrm{h} \sim \mathbb{C N}(0,1)$ is channel gain from source to relay node, $d_{s r}$ is the distance between source and relay, $\mathrm{m}$ is path loss exponent and $\eta_{s}^{r}$ is additive white Gaussian noise (AWGN) at relay node. Therefore, total power received by relay node $\mathrm{P}$ is given by

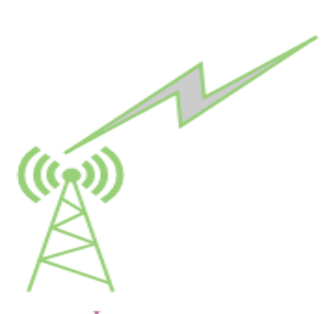

Source S

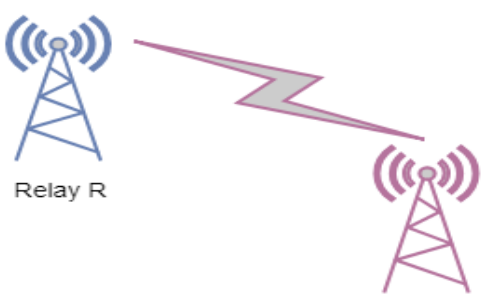

Destination D
Fig 3 System Model

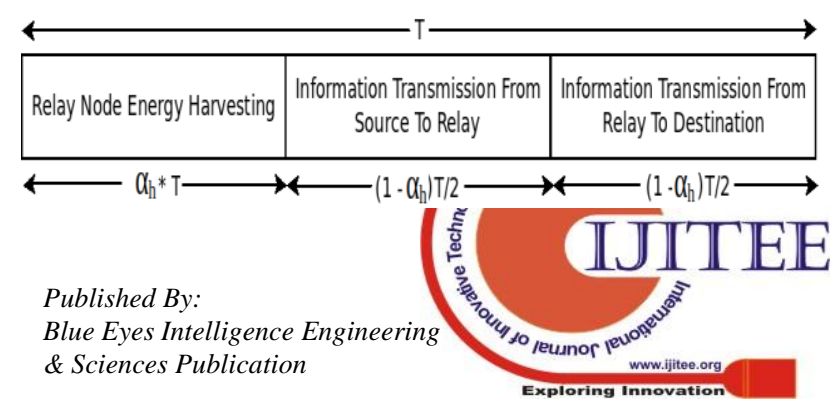


Fig 4 Time division for energy harvesting and information processing

$$
P=\frac{P_{s} h^{2}}{d_{s r}^{m}}
$$

Harvested energy at relay, $\left(\mathrm{E}_{\mathrm{h}}\right)$, during energy harvesting phase, using eq.(2), is calculated as

$$
E_{h}=\frac{\eta P_{s} h^{2}}{d_{s r}^{m}} \alpha_{h} T
$$

where, $\boldsymbol{\eta}$ is energy conversion efficiency. Relay node receive the information from source during the processing phase.

Received signal is converted from RF to baseband. The signal after down conversion at relay node can be expressed as

$$
\mathbb{S}_{r}^{r}=\frac{\sqrt{P_{s}} h s}{\sqrt{d_{s r}^{m}}}+n_{r}^{s}+n_{r}^{c}
$$

where, $n_{\gamma}^{C}$ is additive conversion noise. Before retransmitting signal, relay node amplifies the received signal. Hence, information transmitted by relay is $S_{\%}^{t}$ which is given by

$$
S_{r}^{t}=\frac{\sqrt{P_{r}} \mathbb{S}_{r}^{r}}{\sqrt{\frac{P_{s} h^{2}}{d_{s r}^{m}}+\left(\sigma_{r}^{s}\right)^{2}+\left(\sigma_{r}^{c}\right)^{2}}}
$$

Where $P_{r}$ is transmitted power from relay to destination. There is power constraint at relay node which is represented by denominator factor of eq. 5 . We can see that power constraint factor of relay node can be obtained using power of received signal. By replacing the variance of $n_{r}^{c}$ and $n_{r}^{s}$ with combined variance given below $\sigma_{r}^{2} \triangleq\left(\sigma_{r}^{s}\right)^{2}+\left(\sigma_{r}^{c}\right)^{2}$ eq. 5 can be expressed as

$$
S_{r}^{t}=\frac{\sqrt{P_{r}} \mathbb{S}_{r}^{r}}{\sqrt{\frac{P_{s} h^{2}}{d_{s r}^{m}}+\sigma_{r}^{2}}}
$$

Signal received at the destination node, $S_{\mathbb{d}}^{r}$ is given as

$$
S_{d}^{r}=\frac{g S_{r}^{t}}{\sqrt{d_{r d}^{m}}}+n_{d}^{s}+n_{d}^{c}
$$

where $g \sim \mathbb{C N}(0,1)$ is channel gain from relay to destination. $n_{d}^{s}$ and $n_{d}^{c}$ are antenna and conversion AWGNs at the destination node respectively. Substituting the $S_{r}^{t}$ from eq. (5), into eq. (7), the signal received at destination node is

$$
S_{d}^{r}=\frac{g \mathbb{S}_{r}^{r}}{\sqrt{d_{r d}^{m}} \sqrt{P_{s} h^{2}+d_{s r}^{m}\left(\sigma_{r}^{2}\right)}}+n_{d}^{s}+n_{d}^{c}
$$

$$
S_{d}^{r}=\frac{\sqrt{P_{r} P_{s}} g h s}{\sqrt{d_{r d}^{m}} \sqrt{P_{s} h^{2}+d_{s r}^{m}\left(\sigma_{r}^{2}\right)}}+\frac{\sqrt{P_{r} d_{s r}^{m}} g n_{r}}{\sqrt{d_{r d}^{m}} \sqrt{P_{s} h^{2}+d_{s r}^{m}\left(\sigma_{r}^{2}\right)}}+n_{d}
$$

where $n_{r} \triangleq n_{r}^{c}+n_{r}^{s}$ and $n_{d} \triangleq n_{d}^{c}+n_{d}^{s}$ are combined AWGNs at relay and destination respectively. Communication between relay and destination node take place for $\left(1-\alpha_{h}\right) T / 2$ time period. Hence, from eq. (3), we can derive that $P_{F}$ is also calculated as given in eq. (10)

$$
P_{r}=\frac{E_{h}}{\left(1-\alpha_{h}\right) T / 2}
$$

and it can be further simplified using eq. (3) as given below

$$
P_{r}=\frac{2 \eta P_{s} h^{2} \alpha_{h}}{d_{s r}^{m}\left(1-\alpha_{h}\right)}
$$

Using eq. (9) and (11), we can say that the destination receives signal as given below

$$
\begin{aligned}
& S_{d}^{r}=\frac{\sqrt{2 \eta \alpha_{h}} P_{s} g h^{2} s}{\sqrt{d_{r d}^{m} d_{s r}^{m}\left(1-\alpha_{h}\right)} \sqrt{P_{s} h^{2}+d_{s r}^{m}\left(\sigma_{r}^{2}\right)}} \\
& +\frac{\sqrt{2 \eta \alpha_{h} P_{s}} g h n_{r}}{\sqrt{d_{r d}^{m}\left(1-\alpha_{h}\right)} \sqrt{P_{s} h^{2}+d_{s r}^{m}\left(\sigma_{r}^{2}\right)}}+n_{d}
\end{aligned}
$$

\section{Optimization}

The main objective is to optimize the transmitted power by source, but we need to optimize e taking the QoS (quality of service) into consideration. Here, minimum energy required by destination is taken as QoS. In this section, we will optimize the transmitted power (Ps) to maximize the energy efficiency. Form the eq. (12), we can extract the signal part and the noise part of received signal at destination.

Signal Part $=\frac{\sqrt{2 \eta \alpha_{h}} P_{s} g h^{2} s}{\sqrt{d_{r d}^{m} d_{s r}^{m}\left(1-\alpha_{h}\right)} \sqrt{P_{s} h^{2}+d_{s r}^{m}\left(\sigma_{r}^{2}\right)}}$

Noise Part $=\frac{\sqrt{2 \eta \alpha_{h} P_{s}} g h n_{r}}{\sqrt{d_{r d}^{m}\left(1-\alpha_{h}\right)} \sqrt{P_{s} h^{2}+d_{s r}^{m}\left(\sigma_{r}^{2}\right)}}+n_{d}$

Using eq. (13) and (14), the signal-to-noise ration $\left(S N R_{d}\right)$,

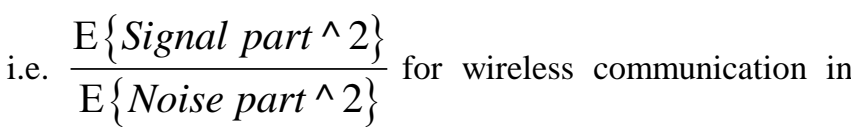
IoT at the destination node can be expressed as

Now we finally substitute $\mathbb{S}_{r}^{r}$ from eq. (4) into eq. (8),

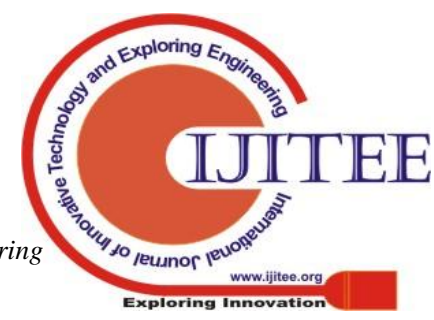




$$
\begin{gathered}
S N R_{d}=\frac{\frac{2 \eta \alpha_{h} P_{s}^{2} g^{2} h^{4}}{\frac{d_{r d}^{m} d_{s r}^{m}\left(1-\alpha_{h}\right)\left(P_{s} h^{2}+d_{s r}^{m}\left(\sigma_{r}^{2}\right)\right)}{2 \eta \alpha_{h} P_{s} g^{2} h^{2} \sigma_{r}^{2}}}}{\operatorname{dNR}_{r d}^{m}\left(1-\alpha_{h}\right)\left(P_{s} h^{2}+d_{s r}^{m}\left(\sigma_{r}^{2}\right)\right)}+\sigma_{d}^{2} \\
2 \eta \alpha_{h} P_{s} g^{2} h^{2} d_{s r}^{m} \sigma_{r}^{2}+d_{r d}^{m} d_{s r}^{m}\left(1-\alpha_{h}\right) P_{s}^{2} h^{2} \sigma_{d}^{2}+d_{s r}^{2 m} d_{r d}^{m} \sigma_{r}^{2} \sigma_{d}^{2}\left(1-\alpha_{h}\right)
\end{gathered}
$$

So, using eq. (11) and (16), the problem to be optimized is formulated to maximize the energy efficiency of IoT system constrained by maximum SNR value and minimum energy harvested by relay node. The formulated problem is given as

$$
\begin{aligned}
& \max _{P_{s}} \frac{\log _{2}\left(1+S N R_{d}\right)}{a P_{s}+b}, \\
& \text { s.t. } S N R_{d} \leq \gamma_{t h r} \text { andP }_{r} \geq \beta_{t h r},
\end{aligned}
$$

where $\beta_{t h r}$ and $\gamma_{t h r}$ are threshold values and represent the minimum value of energy harvested by the relay node and maximum value of SNR at destination node respectively. The optimized solution be achieved by using Particle Swarm Optimization technique (PSO).

\section{A. PSO Approach}

Kennedy and Eberhart [34] proposed the Meta heuristic population based approach which depends on the social behavior of flocking birds and fishes named as "Particle Swarm Optimization (PSO)". PSO [34] works as illustrated in Algorithm 1.

Algorithm 1: Particle Swarm Optimization

1: Initialize the population of particles (random values of transmitted energy) with random positions and velocities in $\mathrm{D}$ dimensions

2: Initialize the pBest for each particle and gBest

3: for $\mathrm{i}=1$ to MaxIt do

4: Evaluate each particle for its objective function

(energy efficiency) and updates it's pBest

5: Update the gBest

6: for each particle do

7: Update velocity using equation 18

8: Update new position using equation 19

9:end for

10: end for

11: return gBest

This algorithm has been applied successfully to many optimization problems. As PSO is population based search algorithm, so each individual particle in the population presents a solution in D dimensions. Every particle has knowledge of personal as well as global best of all the particle so that it can move towards the optimal directions for convergence. The following equations are used to update the position of particle:

$$
v_{i j}=w^{*} v_{i j}+c_{1} * r_{1} *\left(p_{i j}-x_{i j}\right)+c_{2} * r_{2} *\left(p_{g j}-x_{i j}\right)
$$

$$
x_{i j}=x_{i j}+v_{i j}
$$

Equation 18 is used to update the velocity of particle using to personal and global best. Using this updated velocity, new position of particle is updated using equation 19. Here $c_{1}, c_{2}$, $\mathrm{r}_{1}, \mathrm{r}_{2}$ and $\mathrm{w}$ are algorithm parameters.

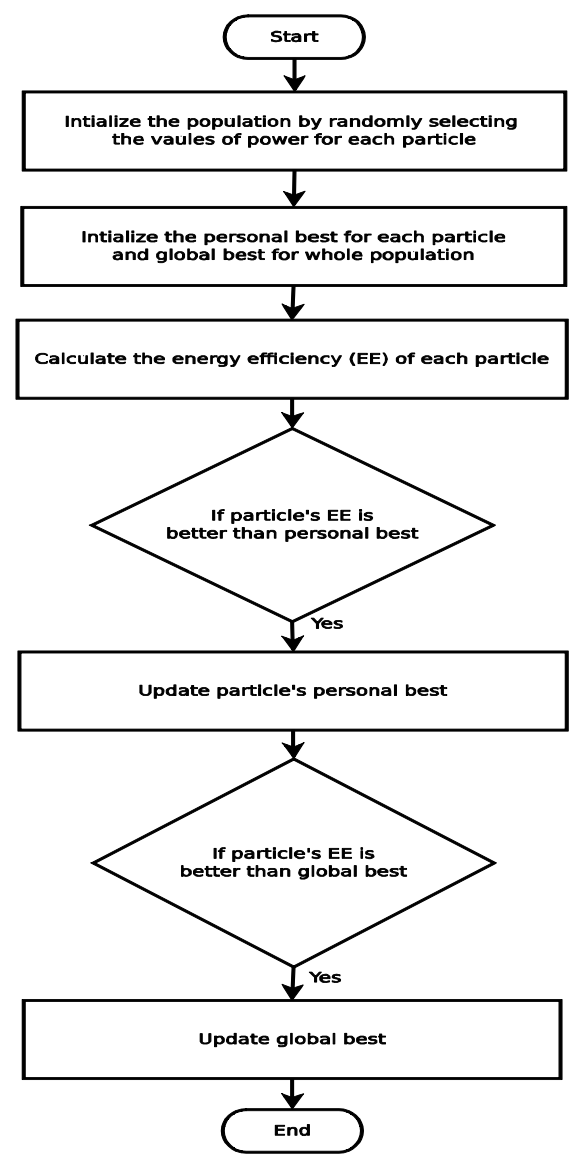

Fig 5 Proposed Method using PSO

In our case, we have to find out the optimized solution for equation 17. PSO is used to find out the value of Ps at which the equation 17 gives optimized value. An individual particle represents values of Ps. So in this case, solution is one dimensional. Fig 5 illustrates the procedure of proposed work using PSO.

In our case, population is initialized by randomly selecting the value of power transmitted from the source node. Initially personal best value of energy efficiency for each particle and

global best value are assigned infinite. In each iteration, Energy Efficiency of each particle is calculated and compared with its previous personal best. If it is better than the previous one, then its personal best of particle is updated otherwise it remains unchanged. Similarly, global best value is also updated. In each iteration, values tend to be converged.

\section{Simulation ReSUlts AND ANALYSIS}

The In this section, simulation results are presented. The energy efficiency of dual-hop IoT system is investigated for the optimal value of transmission power (Ps). The optimal values of Ps to maximize the energy efficiency of system is obtained using PSO optimization technique. Noise covariances $\sigma_{r}^{2}$ and

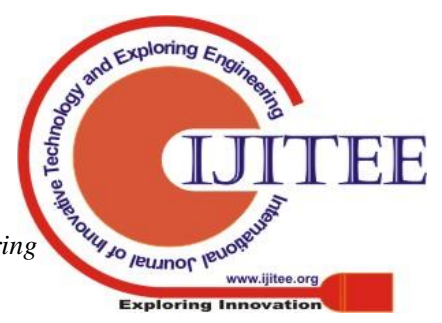


$\sigma_{d}^{2}$ are set to be 1 . The power consumption variables a and b are set to 2 and 10 respectively. And all other parameters except Ps are kept to be 1 just for simplicity.

Experiments are performed over the three different Wi-Fi protocols. These protocols are $802.11 \mathrm{~b}, 802.11 \mathrm{~g}$ and 802.11 $\mathrm{n}$. These three protocols have different values of transmission power. Energy efficiency over these protocols is examined to be maximum by optimizing the power transmitted by the node. Optimization is done by following proposed method as shown in Fig 5. Convergence of energy efficiency and power transmitted using PSO at various iterations are shown in Fig 6 and 7.

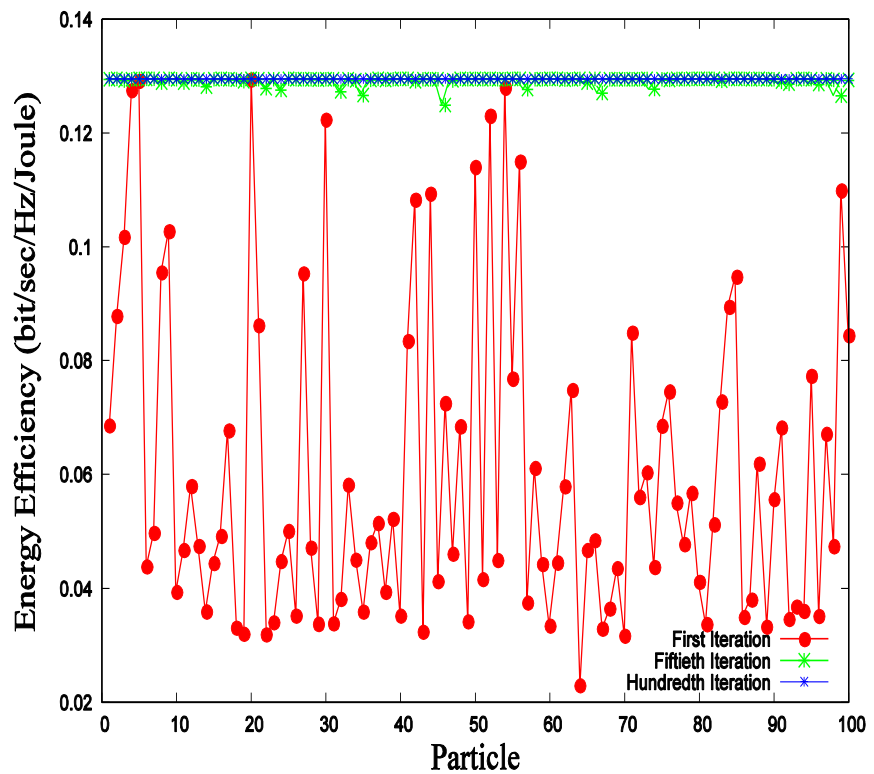

Fig 6 Energy Efficiency for each particle at different iteration

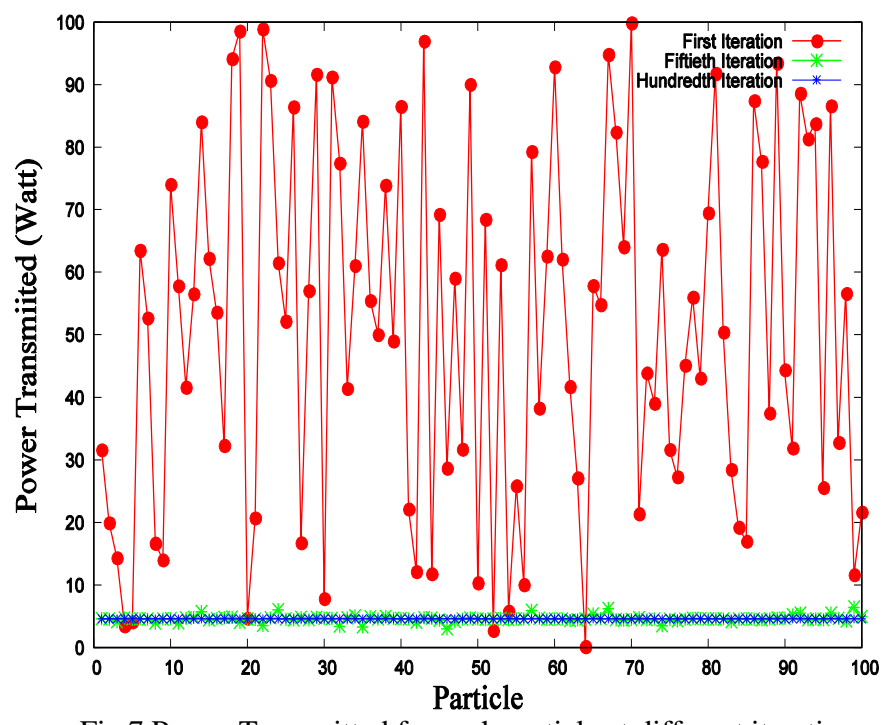

Fig 7 Power Transmitted for each particle at different iteration

It can be seen from Fig 6 that the values of energy efficiency are scattered at the initial iterations and almost converged in hundredth iteration. But in fiftieth iteration, values are more converged than in first and less than in hundredth iteration. So, in each succeeding iteration values move towards the optimum value. Similarly, it happens for the power transmission.
Fig 8 represents the variation of energy efficiency with respect to power transmitted by source node for the protocol 802.11 b. As shown in Fig 8, the energy efficiency decreases with the increase of the transmitted energy. Further, variation of harvested energy by relay node with transmitted power for protocol $802.11 \mathrm{~b}$ is shown in Fig 9. Results indicate that energy harvested by the relay node is directly proportional to the energy transmitted by the source node as illustrated in Fig 9. If the relay node harvests the more energy, then information to be processed by the relay is less and vice versa.

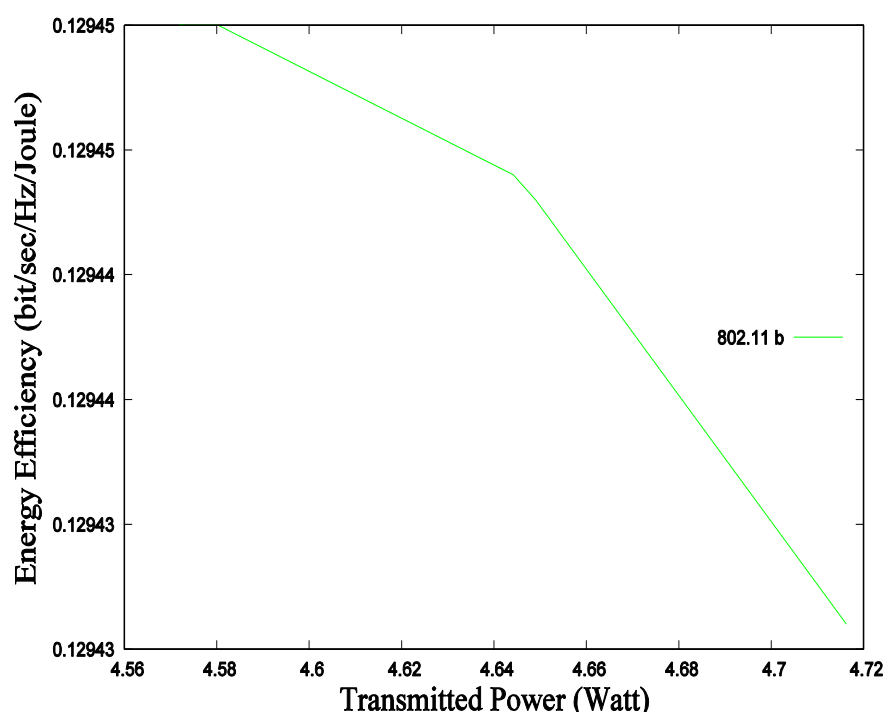

Fig 8 Energy Efficiency vs Power Transmitted

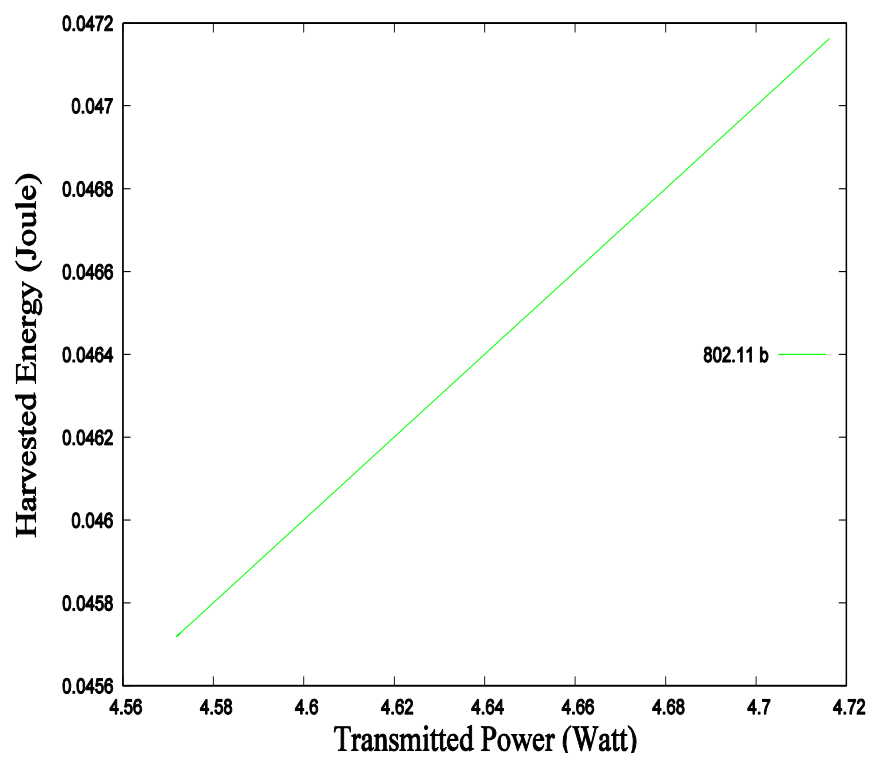

Fig 9 Energy Harvested vs Power Transmitted

For 802.11 b protocol, optimal value of energy transmitted by source is 4.58 Joule and corresponding optimized energy efficiency is 0.12945 . Relay node harvest 0.0458 Joule energy. So it can be concluded from the Fig 8 and 9 that if the energy harvested by relay node increases then energy efficiency at the destination node deceases.

Second protocol used in considered IoT system is $802.11 \mathrm{~g}$. For this protocol, the variation of energy efficiency with respect to

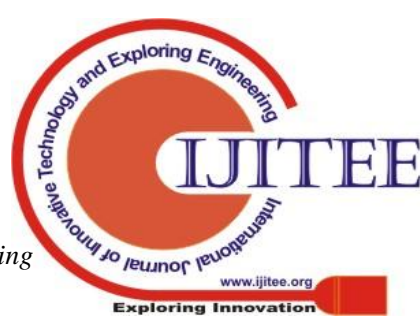


power transmitted by source node is represented in Fig 10 . And Fig 11 plots the variation of harvested energy at relay node for various amount of transmitted energy.

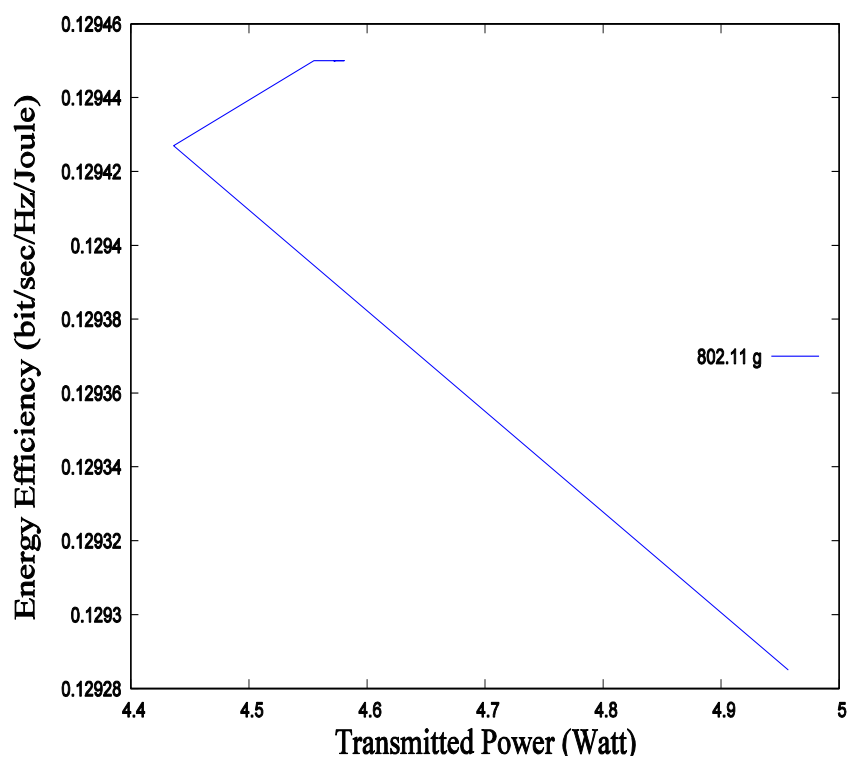

Fig 10 Energy Efficiency vs Power Transmitted

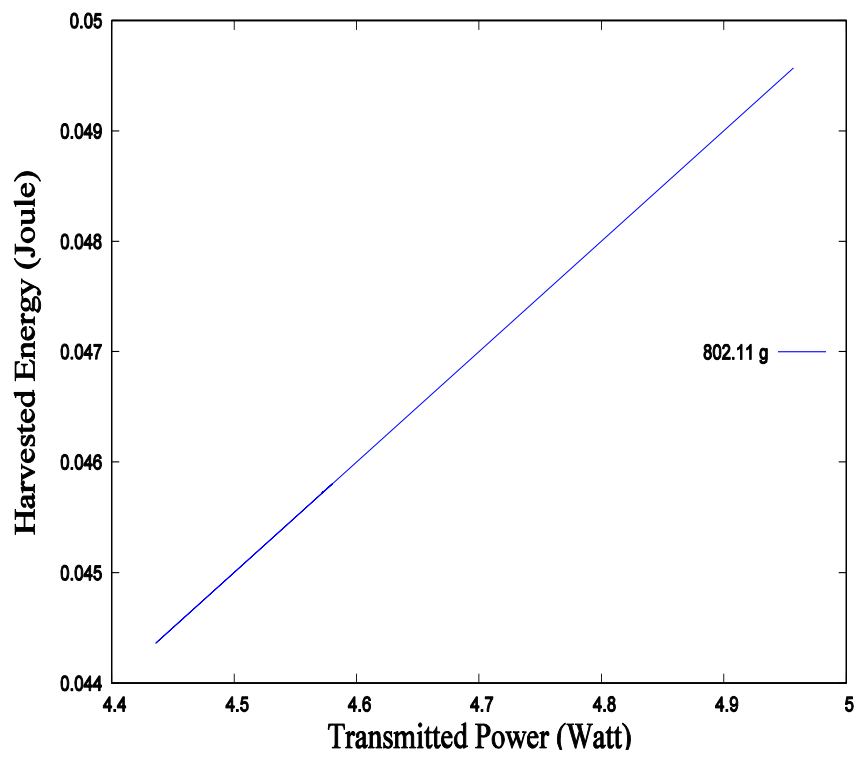

Fig 11 Energy Harvested vs Power Transmitted

Energy efficiency achieved by the proposed method for Wi-Fi protocol $802.11 \mathrm{n}$ is plotted in Fig 12 and energy harvested by relay node for the same protocol is illustrated in Fig 13.

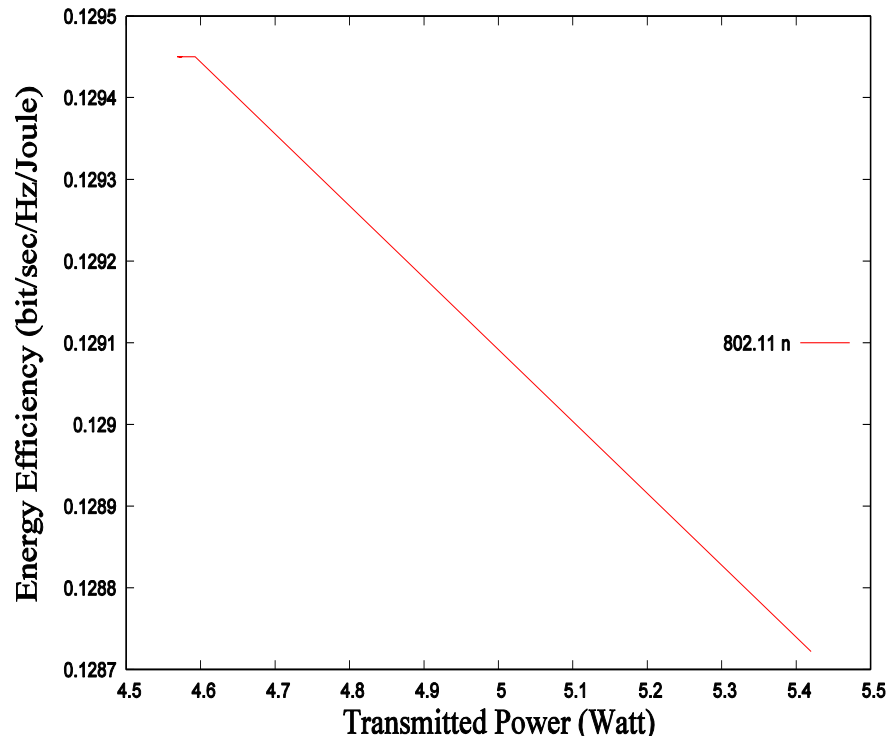

Fig 12 Energy Efficiency vs Power Transmitted

From Fig 8, 10 and 12, it can be seen that all three Wi-Fi protocol $802.11 \mathrm{~b}, 802.11 \mathrm{~g}$ and $802.11 \mathrm{n}$ achieved approximately same energy efficiency. There is very small difference between optimized efficiency for three. Similarly, there is small difference between the energy harvested by the relay node in three protocols.

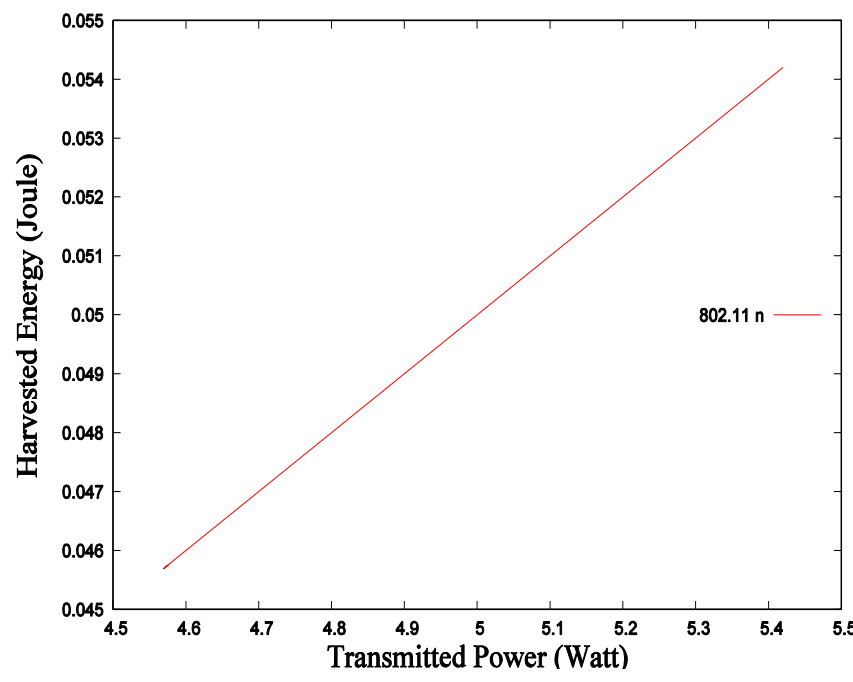

Fig 13 Energy Harvested vs Power Transmitted

The proposed method optimized energy efficiency for all three protocols to approximately same value. Three protocols have different values of transmission power. So their range for the population initialization varies and the initial values of transmitted power are chosen randomly. Hence, achieved energy efficiency for three protocols differ.

\section{CONCLUSION}

The In this paper, a dual-hop relay based Internet of Things system has been analysed for the both energy harvested by relay node and information transfer. A fraction of the transmitted energy is harvested from the RF signals by relay node which can be used to forward the information. Energy efficiency of system

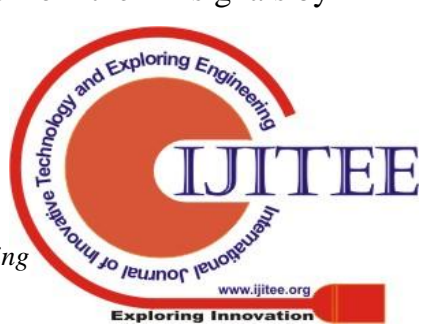


is analyzed towards the maximum using optimized transmitted energy by the source node with the help of PSO. Convergence rate of PSO is very fast. Considered IoT system achieves the optimal energy efficiency at various transmitted energy values and at the same time relay node harvest the minimum energy. Experiments are done using three different Wi-Fi protocols $802.11 \mathrm{~b} / \mathrm{g} / \mathrm{h}$. The optimal value of energy efficiency for each protocol is obtained separately. Energy harvested by the relay

node is directly proportional to power transmitted by source node. As the energy harvested by the relay node increases the energy efficiency at the destination node decreases.

In the future, work can be extended to multi-hop systems. Here, only one parameter is optimized for simplicity. Other parameters like covariances, distances, etc. can also be optimized.

\section{REFERENCES}

1. T. D. Nguyen, J. Y. Khan, D. T. Ngo, Energy harvested roadside ieee 802.15. 4 wireless sensor networks for iot applications, Ad Hoc Networks 56 (2017) 109-121.

2. C. Production, B. Risteska, S. Ss, K. T. Ss, CO (January). doi:10.1016/j.jclepro.2016.10.006.

3. L. Atzori, A. Iera, G. Morabito, The internet of things: A survey, Computer Networks $54 \quad$ (15) (2010) $2787 \quad$ - 2805 doi:https://doi.org/10.1016/j.comnet.2010.05.010.

4. P. Tuwanut, S. Kraijak, A survey on IoT architectures, protocols, applications, security, privacy, real-world implementation and future trends, 11th International Conference on Wireless Communications, Networking and Mobile Computing (WiCOM 2015) (2015) 6-6. doi:10.1049/cp.2015.0714.

5. D. Blaauw, D. Sylvester, P. Dutta, Y. Lee, I. Lee, S. Bang, Y. Kim, G. Kim, P. Pannuto, Y. Kuo, et al., Iot design space challenges: Circuits and systems, in: Symp. VLSI Circuits Dig. Tech. Papers, 2014, pp. 1-2.

6. G. Santucci, et al., From internet of data to internet of things, in: International Conference on Future Trends of the Internet, Vol. 28, 2009.

7. L. Srivastava, Pervasive, ambient, ubiquitous: the magic of radio, in: European Commission Conference From RFID to the Internet of Things,Bruxelles, Belgium, 2006.

8. O. Vermesan, P. Friess, P. Guillemin, S. Gusmeroli, H. Sundmaeker, A. Bassi, I. S. Jubert, M. Mazura, M. Harrison, M. Eisenhauer, et al., Internet of things strategic research roadmap, Internet of Things-Global Technological and Societal Trends 1 (2011) (2011) 9-52.

9. I. Pe na-L_opez, et al., ITU Internet report 2005: the internet of things (2005).

10. IDC, International Data Corporation (IDC) (2018 (accessed Jan 3, 2018)). URL https://www.networkworld.com/article/3244927/inte

11. IDC, International Data Corporation (IDC) website (2016 (accessed June 3, 2018)).

URL ttps://www.idc.com/getdoc.jsp?containerId=prUS41

12. A. Al-fuqaha, S. Member, M. Guizani, M. Mohammadi, S. Member, Internet of Things: A Survey on Enabling Technologies, Protocols, and Applications 17 (4) (2015) 2347-2376. arXiv:jjj. URL http://ieeexplore.ieee.org.proxy.queensu.ca/docum

13. D. Bandyopadhyay, J. Sen, Internet of things: Applications and challenges in technology and standardization, Wireless Personal Communications 58 (1) (2011) 49-69. arXiv:1105.1693, doi:10.1007/s11277-011-0288-5.

14. Rekha, R. Garg, Energy management in wireless sensor networks: A state of art, in: International Conference on Intelligent Data Communication Technologies and Internet of Things, Springer, 2018, pp. 492-499.

15. P. Kamalinejad, C. Mahapatra, Z. Sheng, S. Mirabbasi, V. C. M. Leung, Y. L. Guan, Wireless energy harvesting for the Internet of Things, IEEE Communications Magazine 53 (6) (2015) 102-108.

16. E. Kordetoodeshki, A. Hassanzadeh, An ultra-low power, low voltage dc-dc converter circuit for energy harvesting applications, AEU-International Journal of Electronics and Communications 98 (2019) 8-18.

17. M. Gorlatova, J. Sarik, G. Grebla, M. Cong, I. Kymissis, G. Zussman, Movers and shakers: Kinetic energy harvesting for the internet of things, in: ACM SIGMETRICS Performance Evaluation Review, Vol. 42, ACM, 2014, pp. 407-419.

18. H. G. Lee, N. Chang, Powering the iot: Storageless and converter-less energy harvesting, in: Design Automation Conference (ASP-DAC), $201520^{\text {th }}$ Asia and South Paci_c, IEEE, 2015, pp. 124-129.

19. M. Babar, A. Rahman, F. Arif, G. Jeon, Energy harvesting based on internet of things and big data analytics for smart health monitoring, Sustainable Computing: Informatics and Systems doi:10.1016/j.suscom.2017.10.009.

URL http://dx.doi.org/10.1016/j.suscom.2017.10.009

20. B. Vaisband, E. G. Friedman, Heterogeneous 3-D ICs as a platform for hybrid energy harvesting in IoT systems, Future Generation Computer Systems 87 (2018) 152-158. doi:10.1016/j.future.2018.04.092.

21. H.-S. Nguyen, T.-S. Nguyen, M. Voznak, Relay selection for swipt: Performance analysis of optimization problems and the trade-off between ergodic capacity and energy harvesting, AEU-International Journal of Electronics and Communications 85 (2018) 59-67.

22. S. Bagchi, J. Y. Siddiqui, Throughput optimization using availability analysis based spectrum sensing for a cognitive radio, AEU-International Journal of Electronics and Communications 85 (2018) 12-22.

23. J. Guo, H. Hu, X. Da, J. Liu, W. Li, Optimization of energy efficiency for cognitive radio with partial rf energy harvesting, AEU-International Journal of Electronics and Communications 85 (2018) 74-77.

24. S. Kitazawa, H. Ban, K. Kobayashi, Energy harvesting from ambient rf sources, in: Microwave Workshop Series on Innovative Wireless Power Transmission: Technologies, Systems, and Applications (IMWS), 2012 IEEE MTT-S International, IEEE, 2012, pp. 39-42.

25. X. Lu, P. Wang, D. Niyato, D. I. Kim, Z. Han, C. Engineering, Wireless Networks with RF Energy Harvesting : A Contemporary Survey 17 (2) (2015) 1-34. arXiv:arXiv:1406.6470v6, doi:10.1109/COMST.2014.2368999.

26. L. R. Varshney, Transporting information and energy simultaneously, in: Information Theory, 2008. ISIT 2008. IEEE International Symposium on, IEEE, 2008, pp. 1612-1616.

27. R. Zhang, C. K. Ho, Mimo broadcasting for simultaneous wireless information and power transfer, IEEE Transactions on Wireless Communications 12 (5) (2013) 1989-2001.

28. X. Zhou, R. Zhang, C. K. Ho, Wireless information and power transfer: Architecture design and rate-energy tradeo_, IEEE Transactions on communications 61 (11) (2013) 4754-4767.

29. A. A. Nasir, X. Zhou, S. Durrani, R. A. Kennedy, Relaying protocols for wireless energy harvesting and information processing, IEEE Transactions on Wireless Communications 12 (7) (2013) 3622-3636.arXiv:1212.5406,doi:10.1109/TWC.2013.062413.122042.

30. K. Tang, R. Shi, J. Dong, Throughput analysis of cognitive wireless acoustic sensor networks with energy harvesting, Future Generation Computer Systems.

31. S. Tariq, K. Won, S. Faraz, M. Young, Ad Hoc Networks Throughput analysis of two-way relay networks with wireless energy harvesting capabilities 53 (2016) 123-131.

32. B. Ji, K. Song, C. Li, W.-p. Zhu, L. Yang, Energy harvest and information transmission design in internet-of-things wireless communication systems, AEU-International Journal of Electronics and Communications 87 (2018) 124-127.

33. B. C. Nguyen, T. M. Hoang, P. T. Tran, Performance analysis of full-duplex decode-and forward relay system with energy harvesting over nakagami-m fading channels, AEU-International Journal of Electronics and Communications 98 (2019) 114-122.

34. R. Poli, J. Kennedy, T. Blackwell, Particle swarm optimization, Swarm intelligence 1 (1) (2007) 33-57.

\section{AUTHORS PROFILE}

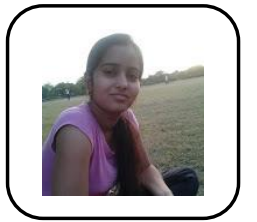

Rekha received her B.Tech degree in Computer Science and Engineering from Deenbandhu Chhotu Ram University of Science and Technology, Murthal, Haryana in 2014. She received her Master's degree in Computer Engineering from Malaviya National Institute of Technology, Jaipur in 2016 Currently, she is pursuing $\mathrm{PhD}$ in the area of Energy Management in Internet of Things from National Institute of Technology, Kurukshetra, India. 
Ritu Garg received B.Tech degree in Computer Science from Punjab Technical University, Jalandhar, India in 2001. She received M.Tech in the area of Computer Science from Kurukshetra University, Kurukshetra in 2006. She received her $\mathrm{Ph} . \mathrm{D}$ in the area of Resourse Management in Grid Computing from National Institute of Technology, Kurukshetra, India. Currently, she is Assistant Professor in the Department of Computer Engineering in National Institute of Technology, Kurukshetra, India. Her research interests include Resource management in Grid/Cloud Computing, Evolutionary Computing, Internet of things, Energy Harvesting, security of IOT etc. 